\title{
Excitonic Magnetoabsorption of Cylindrical Quantum Disks
}

\author{
P. SChillak* And G. CzajkOWski \\ University of Technology and Life Sciences, Bydgoszcz, Poland
}

\begin{abstract}
We show how to compute the optical functions (the complex magnetosusceptibility, dielectric function, magnetoreflection) for semiconductor quantum disks exposed to a uniform magnetic field in the growth direction, including the excitonic effects. The method uses the microscopic calculation of nanostructure excitonic wave functions and energy levels, and the macroscopic real density matrix approach to compute the electromagnetic fields and susceptibilities. The electron-hole screened Coulomb potential is adapted and the valence band structure is taken into account in the cylindrical approximation, thus separating light- and heavy-hole motions. The confinement potentials are taken as step-like both in the $z$ and in-plane directions. Numerical calculations have been performed for $\mathrm{In}_{0.55} \mathrm{Al}_{0.45} \mathrm{As}$ (disk)/ $\mathrm{Al}_{0.35} \mathrm{Ga}_{0.65} \mathrm{As}$ (barrier) and $\mathrm{InP} / \mathrm{GaP}$ disks and the results are in a good agreement with the available experimental data.
\end{abstract}

PACS: 78.67.Hc, 73.22.Dj, 73.21.La, 71.35.Ji

\section{Basic equations}

In the effective mass approximation the exciton is treated as a hydrogen-like atom, where the electron and the hole interact via the Coulomb potential screened by the semiconductor dielectric constant. In typical II-VI and III-V semiconductors (GaAs, for example) the dielectric constant is large and the Wannier-Mott excitons occur, having large Bohr radius and small binding energy (a few meV). In bulk semiconductors the relative and the center-of-mass motion of the electron-hole pair separate and the excitonic energy levels follow the Rydberg formula $-R^{*} / n^{2}$. In semiconductor nanostructures the exciton is squeezed and its binding energy increases even by an order of magnitude. Due to confinement effects accompanied by the $\mathrm{e}-\mathrm{h}$ Coulomb interaction, the separation of the relative- and center-of-mass motion is not possible which makes the calculation of the energy levels very difficult.

On the other hand, potential applications of semiconducting nanostructures in novel optoelectronic devices make the determination of the excitonic energies and resulting optical properties important, since the excitonic resonances occur in the mostly used visible excitation region. In consequence, there is a motivation for developing methods of calculation of the excitonic states, wave functions and the resulting optical properties. Here we consider semiconductor quantum dots of cylindrical symmetry, where the carriers move in the dot material in the step-like potentials in all directions. Such dots are also called the quantum disks (for example, [1] for references).

In addition, a constant magnetic field is applied along the symmetry axis. We use the effective-mass-approximation thus obtaining a two-particle Schrödinger equation where the nanostructure Hamiltonian contains the kinetic energy terms, the Coulomb potential and the

* corresponding author; e-mail: psc@utp.edu.pl confinement potentials. The Schrödinger equation refers to a 6-dimensional configuration space and its analytical solution is not known. Also a direct numerical integration, due to the dimensionality, is rather hopeless.

We have recently proposed [2-6] an approximation where the 6-dimensional problem is reduced to a system of 2-dimensional differential equations, for which the numerical solution is available within a finite computer time. The reduction of the dimensionality goes in the following way: first we extract a 4-dimensional subspace (in-plane coordinates), where the one-particle eigenfunctions and eigenvalues can be obtained analytically

$$
\begin{gathered}
\left(-\mu_{\|} \frac{\partial}{\partial z_{\mathrm{e}}} \frac{1}{m_{z \mathrm{e}}} \frac{\partial}{\partial z_{\mathrm{e}}}-\mu_{\|} \frac{\partial}{\partial z_{\mathrm{h}}} \frac{1}{m_{z \mathrm{~h}}} \frac{\partial}{\partial z_{\mathrm{h}}}\right. \\
\left.+H_{\| \mathrm{e}}+H_{\| \mathrm{h}}-\frac{2}{r}+v_{\mathrm{c}}\right) \Psi=\epsilon \Psi,
\end{gathered}
$$

$m_{z \mathrm{e}, \mathrm{h}}$ being the effective masses in the $z$-direction and $\mu_{\|}$ the in-plane e-h reduced mass, $H_{\| j}$ are the one-particle Hamiltonians of the two-dimensional problem in the plane perpendicular to the $z$-axis (including the terms related to the applied magnetic field), $\epsilon$ is the total energy, $v_{\mathrm{c}}$ is the confinement potential, $v_{\mathrm{c}}=\left(V_{\mathrm{e}, \mathrm{conf}}+\right.$ $\left.V_{\mathrm{h}, \text { conf }}\right) / R^{*}$, and $r$ is the relative $\mathrm{e}-\mathrm{h}$ distance. Since the eigenfunctions and eigenvalues of the operators $H_{\|}$are known, we expand the eigenfunction $\Psi$ in terms of them, arriving finally at the system of coupled two-dimensional equations

$$
\begin{aligned}
& \left(-\mu_{\|} \frac{\partial}{\partial z_{\mathrm{e}}} \frac{1}{m_{z \mathrm{e}}} \frac{\partial}{\partial z_{\mathrm{e}}}-\mu_{\|} \frac{\partial}{\partial z_{\mathrm{h}}} \frac{1}{m_{z \mathrm{~h}}} \frac{\partial}{\partial z_{\mathrm{h}}}\right. \\
& \left.+\epsilon_{n_{1} m_{1}}^{(\mathrm{e})}+\epsilon_{n_{2} m_{2}}^{(\mathrm{h})}+v_{\perp \mathrm{c}}\right) f_{\boldsymbol{n}}^{(L)} \\
& +\sum_{\boldsymbol{n}^{\prime}} V_{\boldsymbol{n}^{n^{\prime}}}^{(L)} f_{\boldsymbol{n}^{\prime}}^{(L)}=\epsilon f_{\boldsymbol{n}}^{(L)},
\end{aligned}
$$

where $\epsilon_{n_{1} m_{1}}^{(\mathrm{e})}, \epsilon_{n_{2} m_{2}}^{(\mathrm{h})}$ are the known eigenvalues of the two-dimensional problem for an electron and a hole, respec- 
tively. The matrix elements $V_{n}^{\boldsymbol{n}^{\prime}}\left(z_{\mathrm{e}}, z_{\mathrm{h}}\right)$ of the Coulomb interaction energy are fourfold integrals which can be reduced to triple integrals by changing variables and performing one integration analytically, the index $\boldsymbol{n}$ stands as an abbreviation for four indices, $\boldsymbol{n}=\left\{n_{1}, m_{1}, n_{2}, m_{2}\right\}$. The total angular momentum $L=m_{1}+m_{2}$ is conserved because of the axial symmetry of the problem, so the sum contains only terms with fixed $L$, as marked in the superscripts.

The above equations, transformed into a set of algebraic equations, are then solved numerically, giving the eigenfunctions and eigenvalues. Having the nanostructure eigenfunctions and eigenvalues, we can determine the system optical properties. Various methods can be used: here we choose the real density matrix approach (also called the Stahl approach) which gives the system susceptibility (both real and imaginary part) - other optical functions can be obtained from it $[7,8]$.

\section{Results}

The above described method is appropriate for nanostructures obeying the cylindrical symmetry as, for example, cylindrical quantum dots, quantum wires, quantum rings, and quantum rods, when the static magnetic field is applied along the symmetry axis.

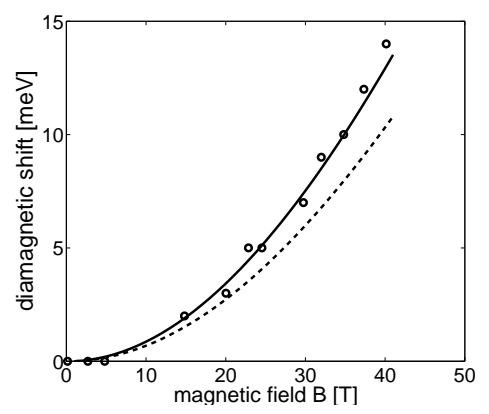

Fig. 1. Diamagnetic shift of the lowest exciton energy calculated from Eqs. (2) for heavy-hole (dashed curve) and light-hole (solid curve). The dots indicate the experimental results by Wang et al. [9].

For the purpose of illustration, we compared our numerical results with the experiments by Wang et al. [9] for the quantum disk with the radius $R=8.95 \mathrm{~nm}$ and the height $d=3.22 \mathrm{~nm}$, as shown in Fig. 1. The material microscopic parameters, typical for $\operatorname{In}_{0.55} \mathrm{Al}_{0.45} \mathrm{As} /$ $\mathrm{Al}_{0.35} \mathrm{Ga}_{0.65} \mathrm{As}$ system, are chosen to be the same as in the paper by Janssens et al. [10]: the dielectric constant $\varepsilon_{\mathrm{b}}=12.71$, electron masses $m_{\mathrm{d}, \mathrm{e}}=0.076 m_{0}, m_{\mathrm{b}, \mathrm{e}}=$ $0.097 m_{0}$, and confinement potentials $V_{0, \mathrm{e}}=258 \mathrm{meV}$, $V_{0, \mathrm{~h}}=172 \mathrm{meV}$. Here d denotes the disk, and b the barrier parameters, respectively.

The dashed curve was calculated for heavy-hole mass $m_{\mathrm{hh}}=0.45 m_{0}$ and one can see that the theoretical result underestimates the measurements. Much better agreement is obtained when we assumed the light-hole mass $m_{\mathrm{lh}}=0.18 m_{0}$, similarly as in Ref. [10].

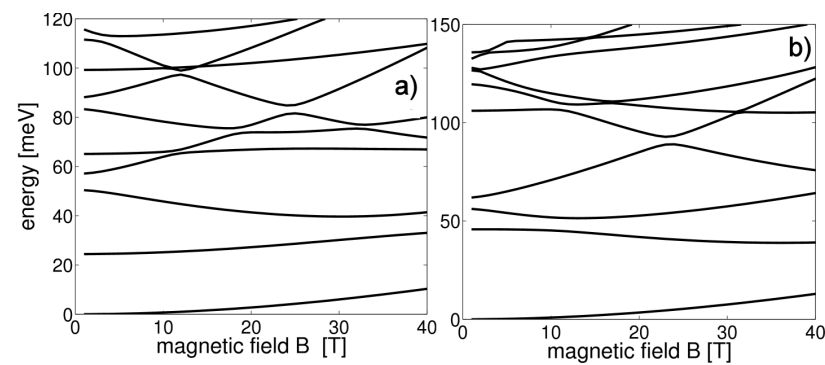

Fig. 2. (a) The relative positions of energy levels as functions of the applied magnetic field for heavy-hole exciton. (b) The same for light-hole exciton. Let us note the anticrossing of all levels in both cases.

The calculated structure of energy levels for the heavy-hole and light-hole excitons, with total angular momentum $L=0$, versus applied magnetic field is presented in Fig. 2. Let us note that the two structures are qualitatively different. The distance between a few lowest levels is determined by the in-plane quantization due to the spatial confinement and by the quantization due to applied magnetic field. We have seen that the Coulomb interaction introduces the anticrossing of all levels and shifts the energies to lower values.

Next, we considered a $\mathrm{InP}$ (dot)/GaP (barrier) quantum disk with the radius $R=8.0 \mathrm{~nm}$ and the height $D=4.0 \mathrm{~nm}$ having in mind the experiments by Dewitz et al. [11]. The electron and the hole effective masses were assumed as $m_{\mathrm{e}}=0.1 m_{0}$, and $m_{\mathrm{h}}=0.35 m_{0}$, respectively, giving the reduced mass $\mu=0.078 m_{0}$. The dielectric constant $\varepsilon_{\mathrm{b}}=14.0$ and the infinite confinement potentials at the dot's boundaries were assumed for an electron and a hole. The deconvolution of photoluminescence spectra enables to determine the magnetic field dependent energies for the ground state and the first excited excitonic state.
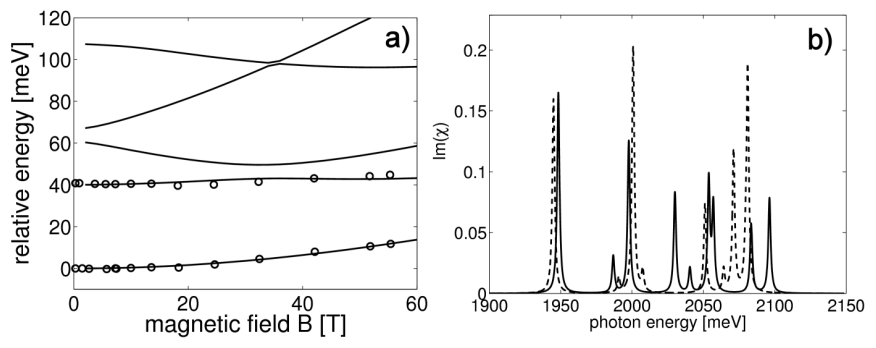

Fig. 3. (a) The measured (circles) [11] and the calculated (full lines) relative positions of energy levels as functions of the applied magnetic field. (b) Imaginary part of the excitonic susceptibility for the $\mathrm{In}_{0.55} \mathrm{Al}_{0.45} \mathrm{As} / \mathrm{Al}_{0.35} \mathrm{Ga}_{0.65} \mathrm{As}$ quantum disk for magnetic field $B=2 \mathrm{~T}$ (dashed line) and $B=20 \mathrm{~T}$ (full line).

The experimental results are marked as circles in Fig. 3. A good agreement of the measured spectra with our calculations can be observed. 
Additionally, our results are consistent with the conclusion of Dewitz et al. [11] that the InP/GaP quantum dot system was of type I. We obtained that, for a weak magnetic field, the energy distance between the first and the second level depends mainly on the assumed disk radius. The diamagnetic shift for the ground state is determined by the electron mass and the hole mass determines the behavior of the first excited level versus magnetic field.

Having the energies and wave functions, we apply the real density matrix approach, which gives the optical functions including magnetoabsorption and the magnetic field dependent dielectric tensor for light- and heavy-hole excitons. In Fig. 3b we present the calculated numerically imaginary part of the magnetosusceptibility for the $\mathrm{In}_{0.55} \mathrm{Al}_{0.45} \mathrm{As} / \mathrm{Al}_{0.35} \mathrm{Ga}_{0.65} \mathrm{As}$ quantum disk.

\section{Conclusions}

We have shown how magnetooptical functions for quantum disks in the excitonic energy region can be computed with a high degree of accuracy. The structure of the optical functions spectra arises from the interplay of three types of quantization: quantization connected with the interaction of the charged particles with external magnetic field, quantization according to the Coulomb interaction and that connected with the finite size of the considered nanostructure.

\section{Acknowledgments}

Numerical computations were carried out at the Academic Computer Center in Gdańsk, Poland.

\section{References}

[1] G. Czajkowski, L. Silvestri, Central Europ. J. Phys. 4, 254 (2006)

[2] P. Schillak, G. Czajkowski, Acta Phys. Pol. A 112, 301 (2007).

[3] P. Schillak, G. Czajkowski, Phys. Status Solidi C 5, 2495 (2008).

[4] P. Schillak, G. Czajkowski, Phys. Status Solidi C 6, 415 (2009).

[5] P. Schillak, G. Czajkowski, Acta Phys. Pol. A 116 , 833 (2009).

[6] P. Schillak, G. Czajkowski, J. Phys. Conf. Series 210 , 012016 (2010).

[7] A. Stahl, I. Balslev, Electrodynamics of the Semiconductor Band Edge, Springer, Berlin 1987.

[8] G. Czajkowski, F. Bassani, L. Silvestri, Riv. Nuovo Cim. C 26, 1 (2003).

[9] P.D. Wang, J.L. Merz, S. Fafard, R. Leon, D. Leonard, G. Medeiros-Ribeiro, M. Oestreich, P.M. Petroff, K. Uchida, N. Miura, H. Akiyama, H. Sakaki, Phys. Rev. B 53, 16458 (1996).

[10] K. L Janssens, F.M. Peeters, V.A. Schweigert, Phys. Rev. B 63, 205311 (2001).

[11] C. v. Dewitz, F. Hatami, M. Millot, J.M. Broto, J. Léotin, W.T. Masselink, Appl. Phys. Lett. 95, 151105 (2009). 